\title{
MULTIPLICATIVE HOMOMORPHISMS OF MATRICES
}

\section{ELIOT CHAMBERLIN AND JAMES WOLFE}

$G$ will denote a system closed under a multiplication. An element $e \in G$ is called an identity if $a e=e a=a$ for every $a \in G$. An element $0 \in G$ is called a null element if $0 a=a 0=0$ for every $a \in G$. Clearly $e$ and 0 are unique if they exist; $e=0$ if and only if $G$ has just one element. A square root of the identity is an element $q \in G$ such that $q^{2}=e$. Let $H \subset G$ be the set consisting of the square roots of the identity in $G$ and the null element if it exists. We assume throughout that the elements of $H$ commute with each other. If $G$ is a ring with identity and without divisors of zero and with ring multiplication as multiplication in $G$, then $H$ consists of $0, e,-e$ and these commute with every element of $G$, for if $q^{2}=e,(q-e)(q+e)=0$ and $q= \pm e$.

$R$ will always denote a ring with identity, and $\mathfrak{M}_{n}$ will denote the set of $n \times n$ matrices with elements in $R$. Let $M_{i}(c), E_{i j}, A_{i j}(c)(i \neq j)$ be the matrices resulting respectively from the identity matrix $I$ by multiplying row $i$ by $c$, interchanging rows $i$ and $j$, and adding row $i$ multiplied by $c$ to row $j$; these will be called elementary matrices. Let $\mathfrak{M}_{n}^{*}$ denote the set of matrices in $\mathfrak{M}_{n}$ which are products of elementary matrices.

For some rings $R, \mathfrak{M}_{n}^{*}=\mathfrak{M}_{n}$; if $R$ is such a ring and $\theta$ is a homomorphism of $R$ onto a ring $R^{\prime}$, then $\mathfrak{M}_{n}^{\prime}{ }^{*}=\mathfrak{M}_{n}^{\prime}$ where the prime refers to matrices with elements in $R^{\prime}$. For $\theta$ induces in a natural way a homomorphism $\theta$ of $\mathfrak{M}_{n}$ onto $\mathfrak{M}_{n}^{\prime}$ (merely let $\theta$ act on each element of the matrix) in which the image of an elementary matrix is elementary. Suppose that a nonnegative integral absolute value $|a|$ is defined in $R$ subject only to the conditions that for every $b \neq 0$ and $a$ in $R, a=b q+r$ and $a=q^{\prime} b+r^{\prime}$ where $|r|,\left|r^{\prime}\right|<|b|$. Then the usual procedure can be used to reduce a matrix in $\mathfrak{M}_{n}$ to diagonal form by left and right multiplications by elementary matrices with inverses; see $[1$, vol. 2 , p. $120 \mathrm{ff}$.]. A diagonal matrix is a product of elementary matrices $M_{i}(c)$ and the inverse of an elementary matrix is elementary if it exists, hence if $R$ has an absolute value as above, $\mathfrak{M}_{n}^{*}=\mathfrak{M}_{n}$. A skew field or field or any euclidean ring admits such an absolute value. If a ring $R$ has such an absolute value and $\beta$ is a homomorphism of $R$ onto a ring $S$, then for $s \in S$ define $|s|=\min |r|$ for $\beta(r)=s$; this gives $S$ an absolute value with the above properties.

A mapping $\Phi$ of $\mathfrak{M}_{n}$ or $\mathfrak{M}_{n}^{*}$ into $G$ such that $\Phi(B C)=\Phi(B) \Phi(C)$ for every $B, C \in \mathfrak{M}_{n}$ or $\mathfrak{M}_{n}^{*}$ respectively, will be called a multiplica-

Received by the editors January 7, 1952. 
tive matrix homomorphism. A mapping $\phi$ of $R$ into $G$ such that $\phi(u v)=\phi(u) \phi(v)$ for every $u, v \in R$ will be called a multiplicative homomorphism. The following simple facts will be used ordinarily without explicit reference.

LEMMA 1. (a) If $\Phi$ is a multiplicative matrix homomorphism of $\mathfrak{M}_{n}$ into $G$, then $\Phi$ confined to $\mathfrak{M}_{n}^{*}$ is a multiplicative matrix homomorphism of $\mathfrak{M}_{n}^{*}$ onto a multiplicatively closed subset of $G$.

If $\Phi$ is a multiplicative matrix homomorphism of $\mathfrak{M}_{n}$ or $\mathfrak{M}_{n}^{*}$ onto $G$, then: (b) Multiplication in $G$ is associative. (c) G has a null element. (d) $G$ has an identity.

The proof is obvious; for example the existence of the null and identity elements in $G$ follows from the existence in $\mathfrak{M}_{n}^{*}$ of the zero and identity matrices $O$ and $I$.

LEMMa 2. Suppose $\Phi$ is a multiplicative matrix homomorphism of $\mathfrak{M}_{n}^{*}$ onto $G$, then :(1) $\left[\Phi\left(E_{i j}\right)\right]^{2}=e .(2) \Phi\left(E_{i j}\right)=\Phi\left(E_{r k}\right) .(3)\left[\Phi\left(M_{i}(-1)\right)\right]^{2}$ $=e$. (4) $\left[\Phi\left(A_{i j}(c)\right)\right]^{2}=e$. (5) $\Phi\left(A_{i j}(c)\right)=\Phi\left(A_{i j}(-c)\right)$. (6) $\Phi\left(A_{i j}(c)\right)$ $=\Phi\left(A_{r k}(c)\right)$. (7) $\Phi\left(E_{i j}\right)=\Phi\left(M_{i}(-1)\right) \Phi\left(A_{i j}(1)\right)$. (8) If $n>2, \Phi\left(A_{i j}(c)\right)$ $=e$. (9) If $n \neq 2$ or if the elements of $H$ commute with every element of $G$, then $\Phi\left(M_{i}(c)\right)=\Phi\left(M_{j}(c)\right)$. (10). If $n>2$ or if $n=2$ and the elements of $H$ commute with every element of $G$, then $G$ is commutative.

The following identities gives these results: (1) $E_{i j} E_{i j}=I$, hence $\Phi\left(E_{i j}\right) \Phi\left(E_{i j}\right)=\Phi(I)=e$. (2) $E_{i j}=E_{r i} E_{r j} E_{r i}$ and $E_{i j}=E_{j i}$ and (1). (3) $M_{i}(-1) M_{i}(-1)=I$. (4) $M_{i}(-1) A_{i j}(c) M_{i}(-1) A_{i j}(c)=I$, hence $\Phi\left(M_{i}(-1)\right) \Phi\left(A_{i j}(c)\right)$ is a square root of $e$ and (4) follows from (3). (5) $A_{i j}(-c)=M_{i}(-1) A_{i j}(c) M_{i}(-1)$ and (3) and (4). (6) $A_{i j}(c)$ $=E_{j k} A_{i k}(c) E_{j k}$ and $A_{i j}(c)=E_{i j} A_{j i}(c) E_{i j}$. (7) $E_{i j}=M_{i}(-1) A_{i j}(1)$ $A_{j i}(-1) A_{i j}(1)$. (8) $A_{i j}(c)=A_{k j}(-1) A_{i k}(-c) A_{k j}(1) A_{i k}(c)$ if $i, j, k$ are distinct; then use (4) and (5). (9) $M_{i}(c)=E_{i j} M_{j}(c) E_{i j}$; if elements of $H$ commute with every element of $G$ then (1) gives the result. If $n=1$, the result is obvious. If $n>2$, using (2), $\Phi\left(M_{1}(c)\right)=\Phi\left(E_{13}\right) \Phi\left(M_{3}(c)\right) \Phi\left(E_{13}\right)$ $=\Phi\left(M_{2}(c)\right)$. Also $\Phi\left(M_{2}(c)\right)=\Phi\left(E_{i j}\right) \Phi\left(M_{1}(c)\right) \Phi\left(E_{i j}\right)$; hence $\Phi\left(M_{1}(c)\right)$ $=\Phi\left(E_{i j}\right) \Phi\left(M_{1}(c)\right) \Phi\left(E_{i j}\right)$ and $\Phi\left(M_{i}(c)\right)=\Phi\left(M_{j}(c)\right)$. (10) If $n \geqq 2$, (9) and the hypotheses of (10) give $\Phi\left(M_{i}(c)\right)=\Phi\left(M_{j}(c)\right)$. But $M_{1}(a) M_{2}(b)$ $=M_{2}(b) M_{1}(a)$, hence all elements of $G$ of the form $\Phi(M)$ commute with each other. Every element of $G$ is a product of elements of the form $\Phi(M)$ and elements of $H$, hence $G$ is commutative if the elements of $H$ commute with every element of $G$. If $n>2$, the last part of the proof of (9) shows that $\Phi(E)$ commutes with every $\Phi(M)$, also $\Phi(A)=e$ by (8). Then every element of $G$ is a product of elements of the forms $\Phi(E)$ and $\Phi(M)$ and these all commute with each other. 
If $\Phi$ is a multiplicative matrix homomorphism of $\mathfrak{M n}_{n}^{*}$ onto $G$ and $n \neq 2$ or the elements of $H$ commute with every element of $G$, then $\Phi\left(M_{i}(c)\right)=\Phi\left(M_{j}(c)\right)$. Define $\phi(c)=\Phi\left(M_{i}(c)\right) ; \phi$ is clearly a multiplicative homomorphism of $R$ into $G$. $\phi$ will be said to be associated with $\Phi$. For $B \in \mathfrak{M}_{n}$ the determinant det $B$ is defined and if $R$ is commutative, det $B C=\operatorname{det} B$ det $C$ for every $B$ and $C$; if $n>1$, this identity implies $R$ is commutative.

TheOREM 1. If $R$ is commutative and $n \neq 2$, every multiplicative matrix homomorphism $\Phi$ of $\mathfrak{M}_{n}^{*}$ onto $G$ is of the form $\Phi(B)=\phi(\operatorname{det} B)$ where $\phi$ is a multiplicative homomorphism of $R$ into $G$ uniquely determined by $\Phi$.

Take $\phi$ to be the multiplicative homomorphism associated with $\Phi$. The result is clear if $n=1$; assume $n>2$. $\Phi\left(M_{i}(c)\right)=\phi(c)$ $=\phi\left(\operatorname{det} \quad M_{i}(c)\right)$. By Lemma 2 part $8, \Phi\left(A_{i j}(c)\right)=e=\phi(1)$ $=\phi\left(\operatorname{det} A_{i j}(c)\right)$ and by Lemma 2 part $7, \Phi\left(E_{i j}\right)=\Phi\left(M_{i}(-1)\right)$ $=\phi(-1)=\phi\left(\operatorname{det} E_{i j}\right)$. Hence $\Phi(B)=\phi(\operatorname{det} B)$ for any elementary matrix, consequently for any matrix in $\mathfrak{M}_{n}^{*}$. If $\Phi(B)=\psi(\operatorname{det} B)$ for every $B \in \mathfrak{M}_{*}^{*}, \psi \equiv \phi$ since $\psi(c)=\psi\left(\operatorname{det} M_{i}(c)\right)=\Phi\left(M_{i}(c)\right)=\phi(c)$.

COROLlaRY. If $F$ is a commutative multiplicative system or a ring without divisors of zero, and if $R$ is a field and $\Phi$ is a multiplicative matrix homomorphism of $\mathfrak{M}_{n}(n \neq 2)$ into $F$, then $\Phi=\phi(\mathrm{det})$ where $\phi$ is a multiplicative homomorphism of $R$ into $F ; \Phi(B)=\Phi(O)$ if $\operatorname{det} B=0$. If $F=R$ and $\Phi\left(M_{1}(c)\right) \equiv c, \Phi=\operatorname{det}$.

For if $F$ is commutative or a ring without divisors of zero, every multiplicatively closed subsystem of $F$ is a system of type $G$. Then Lemma 1 and Theorem 1 give the result.

We shall use $G^{*}$ to denote a system $G$ with the properties: (i) The elements of $H$ commute with every element of $G$. (ii) If $a b=0, a=0$ or $b=0$. (iii) If $q \in H$ and $q a=a$ for some $a \neq 0$, then $q=e$. A ring without divisors of zero, under multiplication, and a group with a null element adjoined are examples of systems $G^{*}$. In a system $G^{*}$, $p=q$ if $p, q \in H$ and $p a=q a$ for some $a \neq 0$.

A multiplicative matrix homomorphism $\Omega$ of $\mathfrak{M}_{2}^{*}$ into $G^{*}$ will be called simple if $\Omega$ maps $\mathfrak{M}_{2}^{*}$ into $H$, and the associated multiplicative homomorphism $\omega$ maps $R$ into the set $\{0, e\} \subset G^{*}$.

THEOREM 2. If $R$ is commutative and $\Phi$ is a multiplicative matrix homomorphism of $\mathfrak{M}_{2}^{*}$ onto $G^{*}$, then $\Phi(B) \equiv \Omega(B) \phi(\operatorname{det} B)$ where $\phi$ is a multiplicative homomorphism of $R$ into $G^{*}$ and $\Omega$ is simple and vanishes simultaneously with $\phi(\mathrm{det})$. Such $\Omega$ and $\phi$ are uniquely determined by $\Phi$. 
Let $\phi$ be the multiplicative homomorphism associated with $\Phi$. By Lemma 2 parts (1) and (4), $\Phi(E)$ and $\Phi(A)$ are in $H$ and are zero only if $\Phi \equiv 0$, similarly for $\phi(-1)$ and $\phi(1)$. Also $\Phi(M)=\phi(\operatorname{det} M)$, hence for any $B \in \mathfrak{M}_{2}^{*}, \Phi(B)=b \phi(\operatorname{det} B)$ where $b \in H$ and $b$ can be taken to be zero if and only if $\phi(\operatorname{det} B)=0$. Then such $b$ is uniquely determined according to condition (iii) on $G^{*}$; let $\Omega(B)=b$. Then $\Omega(B) \Omega(C) \phi(\operatorname{det} B) \phi(\operatorname{det} \quad C)=\Phi(B) \Phi(C)=\Phi(B C)=\Omega(B C) \phi(\operatorname{det} B)$ $\phi(\operatorname{det} C)$. If $\phi(\operatorname{det} B)$ or $\phi(\operatorname{det} C)$ is zero, $\Omega(B) \Omega(C)=0$ and $\phi(\operatorname{det} B C)$ $=0$ hence $\Omega(B C)=0$. If neither $\phi(\operatorname{det} B)$ nor $\phi(\operatorname{det} C)$ is zero, the product is not zero and $\Omega(B) \Omega(C)=\Omega(B C)$, hence $\Omega$ is multiplicative. $\Omega(M) \in\{0, e\}$, hence $\Omega$ is simple. If $\Phi(B) \equiv \Omega^{\prime}(B) \phi^{\prime}(\operatorname{det} B)$ where $\Omega^{\prime}$ and $\phi^{\prime}$ (det) vanish simultaneously, replacing $B$ by $M_{1}(c)$ shows $\phi^{\prime} \equiv \phi$; clearly then $\Omega^{\prime} \equiv \Omega$.

If $\Phi$ in Theorem 2 is simple, $\Omega=\Phi$. Every multiplicatively closed subset of a ring without divisors of zero is a system of type $G^{*}$, hence Theorem 2 holds for multiplicative matrix homomorphisms $\Phi$ into a ring without divisors of zero. If $\Omega$ is simple and $\psi$ is an arbitrary multiplicative homomorphism of $R$ into $G^{*}$, then $\Psi(B)=\Omega(B) \psi(\operatorname{det} B)$ is a multiplicative matrix homomorphism.

Let $\Omega$ be a simple multiplicative matrix homomorphism, let $\omega$ be the multiplicative homomorphism associated with $\Omega$, and let $\sigma(c)$ $=\Omega\left(A_{12}(c)\right)=\Omega\left(A_{21}(c)\right)$. Clearly $\Omega$ is determined by $\omega$ and $\sigma$; for $\Omega(E)$, see the proof of Lemma 2 part 7 .

Lemma 3. Suppose $\Omega$ is simple and $\omega$ and $\sigma$ are as above, then: (1) $\omega(a b)=\omega(a) \omega(b)$. (2) $\sigma(a+b)=\sigma(a) \sigma(b)$. (3) $\omega(a)=0$ or e. (4) If $\Omega \neq 0$ and $a b=1 \in R$, then $\omega(a)=\omega(b)=e$. (5) $[\sigma(a)]^{2}=e$ if $\Omega \neq 0$. (6) If $\omega(a) \neq 0, \sigma(a r)=\sigma(r)$. (7) If $\sigma(r) \equiv e, \Omega \equiv \omega($ det $)$. (8) $\omega(1+1)=0$ or $\Omega \equiv \omega$ (det).

These facts are derived from the following identities. (1) $M_{1}(a) M_{1}(b)=M_{1}(a b)$. (2) $A_{12}(a+b)=A_{12}(a) A_{12}(b)$. (3) $\Omega$ is simple. (4) If $a b=1, M_{1}(a) M_{1}(b)=I$ and $\Omega(I)=e \neq 0$ since $\Omega \not \equiv 0$. (5) Follows from Lemma 2 part 4. (6) $M_{1}(a) A_{12}(a r)=A_{12}(r) M_{1}(a)$, then use (5) and the properties of $G^{*}$. (7) By Lemma 2 part 7 and by Lemma 3 part $4, \omega(-1)=e$ and $\Omega\left(E_{i j}\right)=\Omega\left(M_{i}(-1)\right) \Omega\left(A_{i j}(1)\right)=e=\omega\left(\operatorname{det} E_{i j}\right)$. Also $\Omega\left(A_{i j}(r)\right)=e=\omega\left(\operatorname{det} A_{i j}(r)\right)$ and $\Omega\left(M_{i}(c)\right)=\omega(c)=\omega\left(\operatorname{det} M_{i}(c)\right)$. Hence $\Omega(B)=\omega(\operatorname{det} B)$ for every $B \in \mathfrak{M}_{2}^{*}$. (8) If $\omega(1+1) \neq 0$, $e$ $=\sigma(r) \sigma(r)=\sigma((1+1) r)=\sigma(r)$ by $(6)$. Then use (7).

THEOREM 3. If $R$ is commutative and $1 / 2 \in R$, then all multiplicative matrix homomorphisms $\Phi$ of $\mathfrak{M}_{2}^{*}$ onto $G^{*}$ are of the form $\psi($ det $)$ where $\psi$ is a multiplicative homomorphism of $R$ into $G^{*}$. 
For by Theorem $2, \Phi(B) \equiv \Omega(B) \phi(\operatorname{det} B)$ where $\Omega$ is simple. Then by Lemma 3 part $4, \Omega \equiv 0$ or $\omega(2)=e$, and by part $8, \Omega \equiv 0$ or $\Omega \equiv \omega(\mathrm{det})$. In either case $\Phi$ is of the form $\psi$ (det).

Theorem 3 holds for multiplicative matrix homomorphisms $\Phi$ into a ring without divisors of zero; this is easily seen from a remark following the proof of theorem 2 .

Let

$$
\begin{aligned}
& P_{1}=\left(\begin{array}{l}
10 \\
01
\end{array}\right), \quad P_{2}=\left(\begin{array}{l}
01 \\
11
\end{array}\right), \quad P_{3}=\left(\begin{array}{l}
11 \\
10
\end{array}\right) \\
& N_{1}=\left(\begin{array}{l}
01 \\
10
\end{array}\right), \quad N_{2}=\left(\begin{array}{l}
10 \\
11
\end{array}\right), \quad N_{3}=\left(\begin{array}{l}
11 \\
01
\end{array}\right)
\end{aligned}
$$

be matrices with elements in $R=\Im_{2}$, the field of integers modulo two. Define $\Omega_{0}\left(P_{i}\right)=e \in G^{*}, \Omega_{0}\left(N_{i}\right)=q \in H \subset G^{*}(q \neq 0, i=1,2,3)$ and $\Omega_{0}(B)=0$ for other $2 \times 2$ matrices with elements in $\Im_{2}$. Computation of the multiplication table for the group of matrices $P_{i}$ and $N_{i}$ shows $\Omega_{0}$ to be a multiplicative matrix homomorphism.

THEOREM 4. If $\mathfrak{M}_{2}$ is the set of $2 \times 2$ matrices over a field $R$, all multiplicative matrix homomorphisms $\Phi$ of $\mathfrak{M}_{2}$ onto $G^{*}$, except the homomorphism $\Omega_{0}$ above, are of the form $\psi(\mathrm{det})$ where $\psi$ is a multiplicative homomorphism of $R$ into $G^{*}$. In particular, if $R$ has more than two elements, $\Phi$ is of the form $\psi(\mathrm{det})$.

By Theorem 2, $\Phi=\Omega \phi(\mathrm{det})$ where $\Omega$ is simple. If either $\Omega$ or $\Phi$ is identically 0 or identically $e$, the result is obvious. Suppose $R$ is a field and neither $\Phi$ nor $\Omega$ is identically 0 or identically $e$. If $a \neq 0$, $\sigma(a)=\sigma(1)$ by Lemma 3 parts 4 and 6 ; hence if there is an $r \in R$ distinct from 0 and $-1, \sigma(1)=\sigma(r+1)=\sigma(r) \sigma(1)$. But $\sigma(1) \neq 0$ by Lemma 3 part 5 , hence $\sigma(r)=e$ by condition (iii) on $G^{*}$, and $\sigma(a)$ $=\sigma(1)=\sigma(r)=e$. By Lemma 3 part $7, \Omega=\omega(\operatorname{det})$, and $\Phi$ $=\omega(\mathrm{det}) \phi(\mathrm{det})$. If $\psi(a)=\omega(a) \phi(a), \Phi=\psi(\mathrm{det})$; clearly $\psi$ is a multiplicative homomorphism since $\omega(a) \in H$ and elements of $H$ commute with every element of $G^{*}$.

If $R$ has no element distinct from 0 and $-1, R=\Im_{2}$. Then Lemma 2 shows that $\Phi\left(N_{2}\right)=\Phi\left(N_{3}\right)$ is in $H$ and is not zero since $N_{2}=A_{12}(1)$ and $N_{3}=A_{21}(1)$. Also, since $-1=+1$ and $N_{1}=E_{12}, \Phi\left(N_{1}\right)=\Phi\left(N_{2}\right)$ $=\Phi\left(N_{3}\right)$ using Lemma 2 part 7. It is also easy to see that $\Phi\left(P_{i}\right)=e$. By Theorem 2, $\Phi(B)=0$ if det $B=0$; hence $\Phi\left(P_{i}\right)=e, \Phi\left(N_{i}\right)=q \in H$ $(q \neq 0, i=1,2,3)$ and $\Phi(B)=0$ for other $B \in M_{2}$. Thus $\Phi$ is of the type $\Omega_{0}$. If $q \neq e, \Phi$ is not of the form $\psi$ (det) since $\operatorname{det} P_{i}=\operatorname{det} N_{j}=1$.

Let $\Im$ be the ring of integers and $\theta: \Im \rightarrow \Im_{2}$ be reduction modulo two 
and let $\Theta$ be the induced homomorphism of integral $2 \times 2$ matrices onto $2 \times 2$ matrices with elements in $\Im_{2}$.

Theorem 5. All multiplicative matrix homomorphisms $\Phi$ of the set of $2 \times 2$ matrices with integral elements onto a system $G^{*}$ are of the form $\Phi(B)=\psi(\operatorname{det} B)$ or $\Phi(B)=\Omega_{0}(\Theta(B)) \psi(\operatorname{det} B)$ where $\Omega_{0}$ is given in Theorem 4 and $\psi$ is a multiplicative homomorphism of $\Im$ into $G^{*}$.

Suppose $\Omega$ is a simple homomorphism of integral $2 \times 2$ matrices and is not of the form $\phi(\mathrm{det})$. Then $\sigma(2 n)=\sigma(n) \sigma(n)=e$ and $\sigma(2 n+1)$ $=\sigma(1)=q \in H, q \neq 0$. Using this $q$, define $\Omega_{0}$ as in Theorem 4, then $\Omega\left(A_{i j}(m)\right)=\Omega_{0}\left(A_{i j}(\theta m)\right)=\Omega_{0}\left(\Theta A_{i j}(m)\right)$. Also $\omega(2 n)=\omega(2) \omega(n)=0$ by Lemma 3 part 8 , and $\Omega\left(M_{i}(c)\right)=\Omega_{0}\left(\Theta M_{i}(c)\right) \omega(c)$ since $\Omega_{0}\left(\Theta M_{i}(c)\right)$ vanishes only if $\Omega\left(M_{i}(c)\right)$ vanishes and otherwise is $e$. Thus for matrices of type $M$ and $A$ (hence for arbitrary matrices), $\Omega(B)$ $=\Omega_{0}(\Theta B) \omega(\operatorname{det} B)$. Then using Theorem $2, \Phi \equiv \psi(\operatorname{det})$ or $\Phi$ is of the form $\Omega_{0}(\Theta) \psi($ det $)$ for some multiplicative homomorphism $\psi$ of $\Im$ into $G^{*}$ and $\Omega_{0}$ of the type mentioned in Theorem 4 .

If $G^{*}$ is the set of integers under multiplication, $H=\{0,1,-1\}$. The only homomorphisms of type $\Omega_{0}$ are (taking $\left.q=1\right) \Omega_{0}^{\prime}\left(P_{i}\right)$ $=\Omega_{0}^{\prime}\left(N_{i}\right)=1, \Omega_{0}^{\prime}(B)=0$ if $B \neq N_{i}, P_{i}$, and $\Omega_{0}^{\prime \prime}\left(P_{i}\right)=1, \Omega_{0}^{\prime \prime}\left(N_{i}\right)=-1$, $\Omega_{0}^{\prime \prime}(B)=0$ if $B \neq N_{i}, P_{i} . \Omega_{0}^{\prime}$ is of the form $\psi(\mathrm{det})$.

CoROLLARY. Every multiplicative matrix homomorphism of integral $2 \times 2$ matrices into $\Im$ is of the form $\psi(\mathrm{det})$ or $\Omega_{0}^{\prime \prime}(\Theta) \psi(\mathrm{det})$ for some multiplicative homomorphism $\psi$ of $\Im$ into $\Im$.

\section{REFERENCE}

1. B. L. van der Waerden, Moderne Algebra, vols. I, II, 1931.

UNTVERSTTY OF UTAB 